\title{
Assessment and management of human health risk from toxic metals and polycyclic aromatic hydrocarbons in urban stormwater arising from anthropogenic activities and traffic congestion
}

\author{
Yukun Ma ${ }^{\text {a,b }}$, An Liu ${ }^{\text {b,c,* }}$, Prasanna Egodawatta ${ }^{\text {b }}$, James McGree ${ }^{\text {b }}$, Ashantha Goonetilleke ${ }^{\text {b }}$ \\ a State Key Laboratory of Urban and Regional Ecology, Research Center for Eco-Environmental Sciences, Chinese Academy of Sciences, 18 Shuangqing Road, Beijing 100085, China \\ b Science and Engineering Faculty, Queensland University of Technology (QUT), GPO Box 2434, Brisbane, 4001, Queensland, Australia \\ c College of Chemistry and Environmental Engineering, Shenzhen University, 518060 Shenzhen, China
}

\section{H I G H L I G H T S}

- Toxic chemical pollutants constrain urban stormwater reuse potential.

- Anthropogenic activities and traffic congestion influence risk from stormwater.

- Quantitative risk model developed incorporating these factors.

- Recommendations for risk mitigation and risk management are identified.

\section{G R A P H I C A L A B S T R A C T}

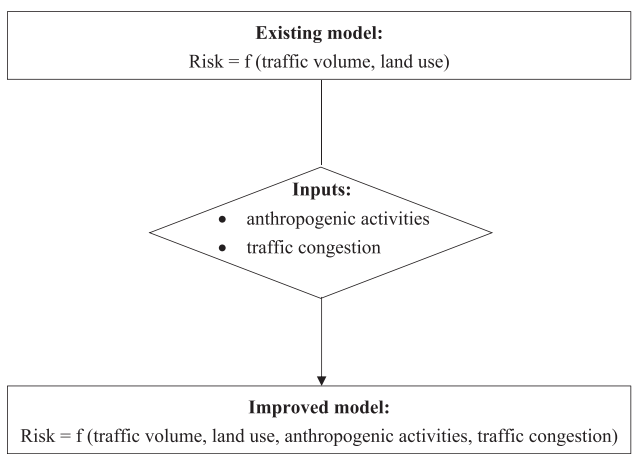

\begin{abstract}
A B S T R A C T
Toxic metals (TMs) and polycyclic aromatic hydrocarbons (PAHs) in urban stormwater pose risk to human health, thereby constraining its reuse potential. Based on the hypothesis that stormwater quality is primarily influenced by anthropogenic activities and traffic congestion, the primary focus of the research study was to analyse the impacts on human health risk from TMs and PAHs in urban stormwater and thereby develop a quantitative risk assessment model. The study found that anthropogenic activities and traffic congestion exert influence on the risk posed by TMs and PAHs in stormwater from commercial and residential areas. Motor vehicle related businesses (FVS) and traffic congestion (TC) were identified as two parameters which need to be included as independent variables to improve the model. Based on the study outcomes, approaches for mitigating the risk associated with TMs and PAHs in urban stormwater are discussed. Additionally, a roadmap is presented for the assessment and management of the risk arising from these pollutants. The study outcomes are expected to contribute to reducing the human health risk associated urban stormwater pollution and thereby enhance its reuse potential.
\end{abstract}

(c) 2016 Elsevier B.V. All rights reserved.

\footnotetext{
* Corresponding author at: College of Chemistry and Environmental Engineering, Shenzhen University, 518060 Shenzhen, China.
}

E-mail address: liuan@szu.edu.cn (A. Liu). 


\section{Introduction}

Urban stormwater runoff carries numerous toxic chemical pollutants generated by anthropogenic activities common to urban areas. Toxic metals (TMs) and polycyclic aromatic hydrocarbons (PAHs) have been identified as among the most important chemical pollutants in urban stormwater due to their toxicity to humans (Sansalone and Buchberger, 1997). TMs can pose potential risk to kidneys, skeleton, nervous system, intestines and stomach (Järup, 2003), while PAHs are commonly carcinogenic to skin, lungs and bladder (Chen and Liao, 2006). Presence of these chemical pollutants reduces the reuse potential of stormwater as an alternative water resource for recreational and potable proposes (Begum et al., 2008). This is primarily due to concentrations of TMs and PAHs commonly being above the threshold levels specified in the relevant guidelines. Liu et al. (2016b) analysed PAHs in stormwater from an urban area in Shenzhen, China and found that the heavy molecular weight PAHs would pose ecological risk to aquatic environments due to high concentrations present in road dust. Herngren (2005) collected wash-off samples in a commercial area in Gold Coast, Australia and analysed the PAHs. It was found that concentrations of benzo[a]pyrene ranged from 0.13 to $0.77 \mathrm{ppm}$ for different wash-off events which exceeded the drinking water guideline value provided by NHMRC\&AWRC (2011) to a significant extent.

In order to manage the human health risk posed by TMs and PAHs in urban stormwater, it is essential to have a methodology to quantitatively assess the potential risks. Previous research has identified that traffic volume (Gunawardena et al., 2013) and land use (Herngren et al., 2006) as the primary factors influencing stormwater quality in relation to TMs and PAHs. Traffic volume is the daily average amount of vehicles using a particular roadway. Ma et al. (2016) used traffic volume and percentage of different land use types as the primary influential factors for developing a quantitative risk assessment methodology.

However, previous research studies have also noted that there are factors other than traffic volume and land use that can influence urban stormwater quality. For example, Lee et al. (2009) and Onderka et al. (2012) found that urban stormwater quality is not only influenced by land use type, but also affected by the spatial configuration of land uses such as the extent, distribution, intensity, and frequency of various anthropogenic uses. Similarly, Liu et al. (2012) noted the significant influence of other anthropogenic activities excluding traffic activities on stormwater quality. As they noted, these anthropogenic activities influence traffic congestion which has been identified as a primary factor which influences stormwater quality. The current study is a further extension of the work undertaken by Ma et al. (2016) cited above. This also applies to the data set used for the current study, which is also a further expansion of original data set used by Ma et al. (2016). However, the inclusion of anthropogenic activities and traffic congestion for undertaking quantitative risk assessment in relation to TMs and PAHs in stormwater is not straightforward due to the qualitative nature of these factors. Consequently, a novel approach is required to include qualitative factors for estimating the risk posed by TMs and PAHs.

This paper presents the outcomes of a wide-ranging study undertaken to: (1) investigate the influence of anthropogenic activities and traffic congestion on the risk posed by TMs and PAHs; (2) enhance the risk model developed by Ma et al. (2016) by taking anthropogenic activities and traffic congestion into consideration; (3) provide recommendations for managing the human health risk from TMs and PAHs in stormwater. It is important to note that the anthropogenic activities identified in this paper mainly refer to specific land use activities such as retail, education, hospitality, catering and vehicle service industries and excludes traffic related activities. The novelty of the research study is the enhancement of the existing model by considering the influence of qualitative factors such as anthropogenic activities and traffic congestion for assessing human health risk posed by TMs and PAHs in stormwater. The outcomes from the study are expected to contribute to strengthening the quantitative assessment and management of the potential human health risk posed by TMs and PAHs present in urban stormwater.

\section{Materials and methods}

\subsection{Study sites}

The study sites were selected from Gold Coast, Australia. Since land use and traffic volume influence the build-up load of pollutants on road surfaces (Liu et al., 2016a), 20 road sites were selected comprising of different land use types and traffic volumes. Road surfaces have been identified as a primary source of TMs and PAHs to urban stormwater (Vaze and Chiew, 2002). The four land use types included commercial, industrial, residential and natural areas, while traffic volumes in the road study sites selected were in the range of 150 to 3000 vehicles per day (VPD). Additionally, as the primary objective of this study was to investigate the influence of anthropogenic activities and traffic congestion on stormwater quality and the associated risk, the variability of these factors was also important for selecting the study sites. Accordingly, the selected road sites exhibited a range of anthropogenic activities and traffic characteristics. Roads with high traffic congestion were identified as the roads with traffic volume not less than 3000 vehicles per day and with traffic lights installed and parking bays. Otherwise, the traffic congestion in the road was considered to be low. Additionally, during the sampling period, the traffic volume leading to congestion was recorded. The selected road study sites are shown in Fig. 1 and Table 1. The land use type, traffic volume, anthropogenic activities and traffic congestion shown in Table 1 are the average characteristics during the day, at the road study sites.

\subsection{Build-up sample collection}

Pollutant build-up samples were collected from the selected road study sites. Although the wash-off process exerts influence on stormwater quality, it was considered relatively less important compared to the build-up process due to the fact that only a fraction of build-up pollutant load is washed off by a single stormwater runoff event (Egodawatta et al., 2007). Accordingly, the risk posed by buildup pollutants was considered as equivalent to the maximum possible risk posed by the TMs and PAHs in stormwater runoff. The build-up samples at a road site were collected in two sampling episodes. Accordingly, 40 build-up samples were collected from the 20 study sites. Each sample collection was undertaken after seven to nine antecedent dry days. This was based on the fact that the build-up load of pollutants on road surfaces asymptotes to an almost maximum value after about seven dry days (Egodawatta and Goonetilleke, 2006). The samples were collected from the road surface from a one-meter-wide plot extending from the kerb to the middle of the road. A dry and wet vacuum method was used for sample collection and the recovery from sampling was above $95 \%$, which was considered as acceptable. The build-up samples were initially collected using a vacuum cleaner. After the dry vacuuming, the plot was moistened with deionised water using a sprayer and the wet samples were vacuumed again into the vacuum cleaner. Further details about the sampling procedure can be found in Gunawardana et al. (2014).

\subsection{Laboratory analysis}

The build-up samples were extracted and analysed for nine TMs and 15 PAHs. The nine TMs included aluminium (Al), cadmium (Cd), hexavalent chromium $(\mathrm{Cr})$, copper $(\mathrm{Cu})$, iron $(\mathrm{Fe})$, lead $(\mathrm{Pb})$, manganese $(\mathrm{Mn})$, nickel (Ni) and zinc ( $\mathrm{Zn})$. The 15 PAHs were, naphthalene (NAP), acenaphthylene (ACY), acenaphthene (ACE), fluorene (FLU), phenanthrene (PHE), anthracene (ANT), fluoranthene (FLA), pyrene (PYR), benz $[a]$ anthracene $(\mathrm{B}[a] \mathrm{A})$, chrysene (CHR), benzo[b]fluoranthene $(\mathrm{B}[b] \mathrm{F})$, benzo[a]pyrene $(\mathrm{B}[a] \mathrm{P})$, indeno(1,2,3-cd)pyrene (IND), 


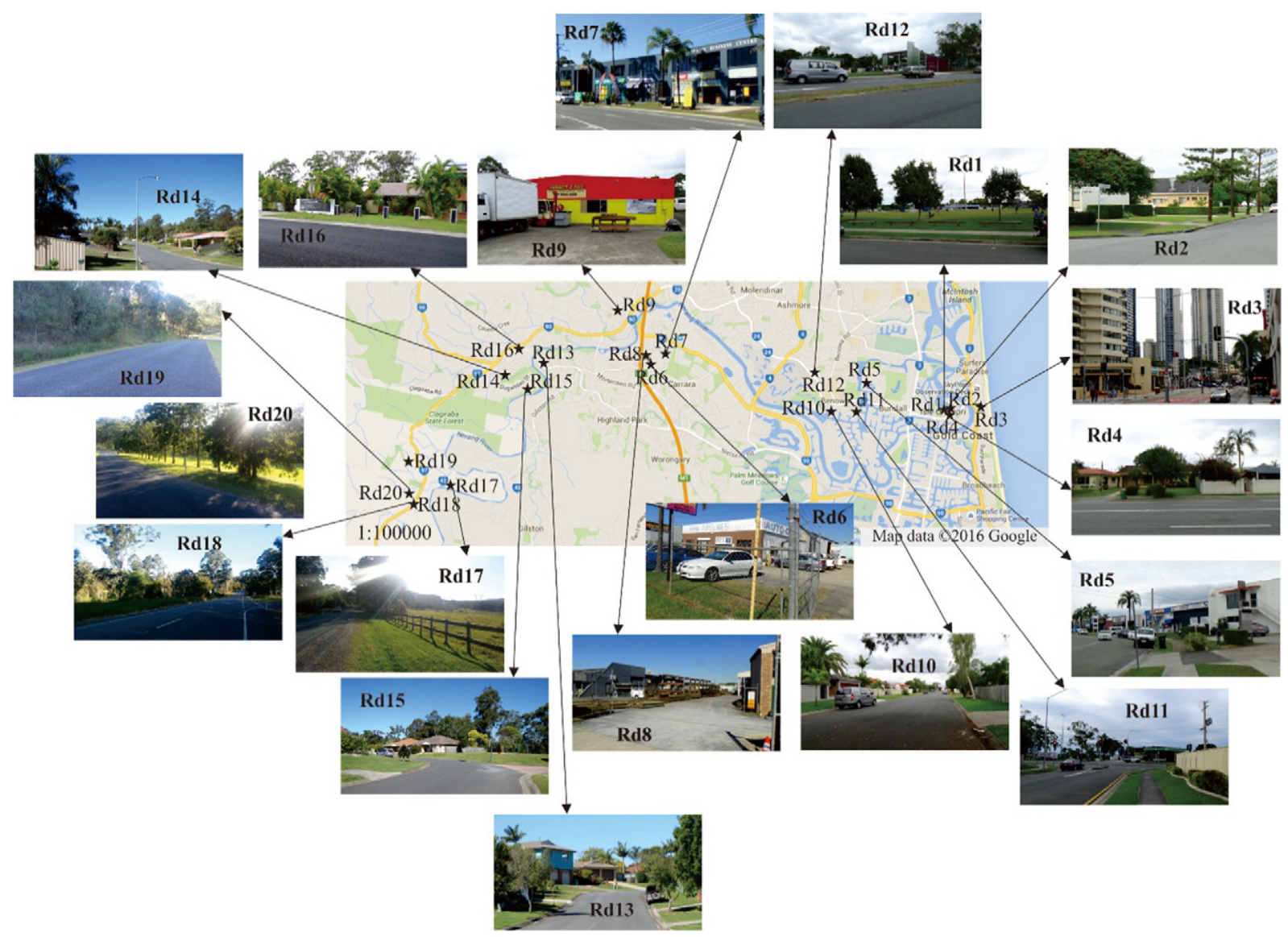

Fig. 1. Study sites.

dibenzo[a,h]anthracene $(\mathrm{D}[a] \mathrm{A})$, and benzo[ghi]perylene $(\mathrm{B}[g] \mathrm{P})$. These species were investigated due to their prevalence in urban stormwater and toxicity to humans (Makepeace et al., 1995). The TMs were extracted using nitric acid digestion according to Method 3030E (USEPA, 1984) and tested using an Agilent 8800 Triple Quadrupole Inductively Coupled Plasma Mass Spectrometer (ICP-MS) (USEPA, 1994). The PAHs were extracted using liquid-liquid extraction and accelerated solvent extraction which met the requirements of Methods USEPA (1984) and USEPA (1996), respectively. The extracts of PAHs were tested using a Shimadzu Gas Chromatograph Mass Spectrometer (GC-MS) TQ8030. Limit of detection (LOD) of TMs and PAHs can be found in Table S1 in the Supplementary Information. As part of quality control and quality assurance, the recovery of the extraction and testing was verified by spiking a known amount of standards into blanks and undertaking the same analysis as the samples.

Table 1

Characteristics of the study sites including land use, traffic volume, anthropogenic activities and traffic congestion.

\begin{tabular}{|c|c|c|c|c|}
\hline $\begin{array}{l}\text { Study } \\
\text { Sites }\end{array}$ & Land Use & Anthropogenic activities & $\begin{array}{l}\text { Daily traffic volume [vehicles per } \\
\text { day] }\end{array}$ & $\begin{array}{l}\text { Traffic } \\
\text { congestion }\end{array}$ \\
\hline $\mathrm{Rd} 1$ & Commercial & Retail, education, catering, hospitality and offices & 750 & Low \\
\hline $\operatorname{Rd} 2$ & Commercial & & 750 & Low \\
\hline $\operatorname{Rd} 3$ & Commercial & & 3000 & High \\
\hline $\mathrm{Rd} 4$ & Commercial & & 3000 & High \\
\hline Rd5 & Commercial & Motor vehicle related businesses & 3000 & High \\
\hline Rd6 & Industrial & Industrial including vehicle repair, pipeline manufacture, steel and aluminium fabrication & 3500 & High \\
\hline Rd7 & Industrial & $\begin{array}{l}\text { Industrial including vehicle repair, painting and panel repair, battery manufacture and } \\
\text { welding }\end{array}$ & 7000 & High \\
\hline Rd8 & Industrial & Industrial including wood work, tile manufacture & 750 & Low \\
\hline Rd9 & Industrial & Industrial including furniture manufacture and plumbing components manufacture & 3500 & Low \\
\hline $\operatorname{Rd} 10$ & Residential & Residential, retail, hospitality catering and education & 500 & Low \\
\hline Rd11 & Residential & & 750 & Low \\
\hline $\mathrm{Rd} 12$ & Residential & Residential and education & 750 & Low \\
\hline Rd13 & Residential & Residential & 500 & Low \\
\hline Rd14 & Residential & & 750 & Low \\
\hline Rd15 & Residential & & 750 & Low \\
\hline $\operatorname{Rd} 16$ & Residential & & 3000 & Low \\
\hline Rd17 & Natural & Very limited anthropogenic activities & 750 & Low \\
\hline Rd18 & Natural & & 1000 & Low \\
\hline Rd19 & Natural & & 150 & Low \\
\hline $\operatorname{Rd} 20$ & Natural & & 150 & Low \\
\hline
\end{tabular}


Deionised water was used as blanks for liquid samples while sea sand was used as blanks for particulate samples. The recovery of TMs and PAHs was found to be within $85 \%-105 \%$ and $75 \%-105 \%$, respectively, which met the requirements of the test methods (USEPA, 1984; USEPA, 1994). The analysis results of TMs and PAHs load in build-up samples are provided in Table S2 and S3 in the Supplementary Information.

\section{Results and discussion}

\subsection{Comparison of risk associated with stormwater from study sites}

Since the risk associated with stormwater is influenced by its quality, the concentration of TMs and PAHs was simulated based on the buildup load of TMs and PAHs according to the procedure discussed in Ma et al. (2016) and the procedure is presented in the Supplementary Information. The risk assessment procedure for TMs and PAHs in stormwater from the study sites is also briefly discussed in the Supplementary Information and detailed information can be found in Ma et al. (2016) and Ma (2016). The risk from TMs was estimated as hazard index (HI) and the risk from PAHs was estimated as incremental lifetime cancer risk (ILCR). In the existing models, HI and ILCR were estimated based on the daily traffic volume (DTV) [vehicles per day] and the percentages of commercial, industrial and residential land use area (C, I and R) around the study site (Eq. (1)). As can be seen in Eq. (1), the specific influence of anthropogenic activities and traffic congestion was not taken into consideration. The risk assessment results are shown in Table S5 and S6 in the Supplementary Information.

Risk $=f(\mathrm{DTV}, \mathrm{C}, \mathrm{I}, \mathrm{R})$

where: DTV - daily traffic volume [vehicles per day];

$\mathrm{C}$, I and R - percentage of commercial, industrial and residential land use areas within $1 \mathrm{~km}$ around the site.

Estimated risk indices of $\mathrm{HI}$ and ILCR related to the road sites were visually compared to investigate their relationship with traffic volume and predominant land use type (Fig. 2). It is clear from Fig. 2 that although both traffic volume and conventional land use types influence the risk significantly, they are not adequate in predicting risks to a satisfactory level. As evident in Fig. 2, stormwater from different road sites within the same land use type and traffic volume can exhibit different risks (for example Rd3, Rd4 and Rd5). This is attributed to factors other than traffic volume and land use specific to those sites (Lee et al., 2009; Liu et al., 2012). This highlights the need for taking into consideration additional factors such as anthropogenic activities and traffic congestion in the surrounding area of the study sites for more accurate assessment of the risk associated with stormwater. It is important to note that although only two samples were collected at each study site, the build-up pollutant loads were assumed to be stable with relatively low variability and is approximate equal to the maximum value as discussed in Section 2.2.

\subsection{Influence of anthropogenic activities and traffic congestion on the risk}

A quantitatively based ranking was undertaken prior to including qualitative factors such as anthropogenic activities and traffic congestion into the risk assessment methodology in order to rank the effects of TMs and PAHs in stormwater on human health risk. This task was undertaken using Preference Ranking Organisation Method for Enrichment Evaluations (PROMETHEE) and the Graphical Analysis for Interactive Assistance (GAIA) method. Both, PROMETHEE and GAIA are increasingly applied in environmental research studies (e.g. Gunawardana, 2011; Gunawardena et al., 2012; Liu et al., 2012). A data matrix needs to be prepared for the PROMETHEE and GAIA analysis. The rows of the data matrix are called actions which represent the objects while the columns are named criteria referring to the variables. PROMETHEE facilitates decision-makers to select the most preferred
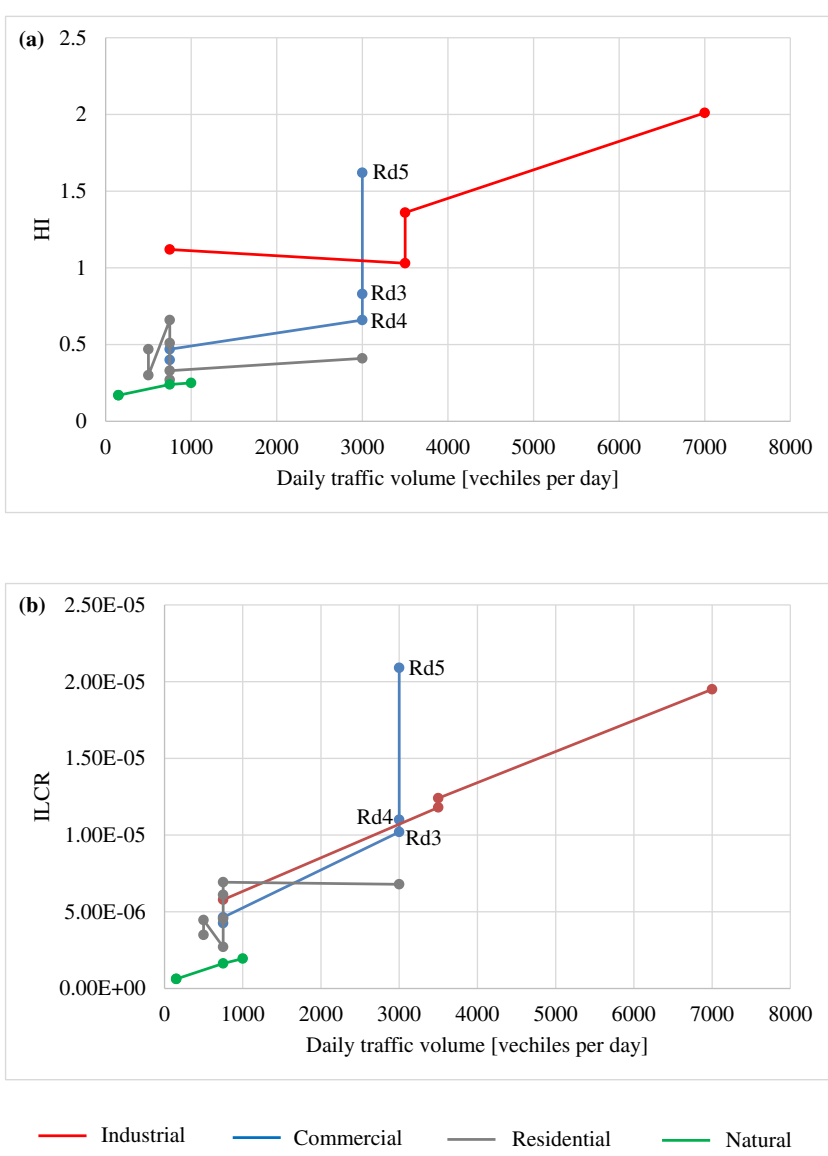

Fig. 2. (a) HI of TMs in stormwater from the study sites; (b) ILCR of PAHs in stormwater from the study sites.

objects according to the most important variables while the GAIA method is the graphical representation of the PROMETHEE analysis outcomes which shows the relationship between the objects and variables. Detailed information about the PROMETHEE and GAIA method can be found in Herngren et al. (2006). To enable PROMETHEE and GAIA analysis, a data matrix containing 20 rows (20 study sites) and 2 criteria (HI and ILCR) were prepared. The analysis outcomes are shown in Fig. 3. According to the ranking results, the risk associated with the study sites could be categorised into different groups.

As evident from Fig. 3, the decision axis Pi is pointed towards the $(+)$ PC1 axis. This indicates that the risk from pollutants in stormwater from the sites in the (+) PC1 axis is higher than those along the (-) PC1 axis. This highlights the fact that the level of risk can be categorised based on sites positioning along the PC1 axis. Accordingly, the study sites were categorised into three groups; (a) high risk, (b) moderate risk and (c) low risk as shown in Fig. 3. According to this categorisation, stormwater generated from the seven road sites present high risk including Rd3, Rd4, Rd5, Rd6, Rd7, Rd8 and Rd9. Furthermore, Rd17, $\operatorname{Rd} 18, \operatorname{Rd} 19, \operatorname{Rd} 20$ are very close to each other and located in the negative PC1 direction. Hence, pollutants in stormwater runoff from these four road sites carry a low risk. The other nine road sites which are located between the high and low risk groups were deemed to present moderate risk to human health. The reasons for these risk levels from pollutants present in stormwater are discussed below.

(a) High risk (Rd3, Rd4, Rd5, Rd6, Rd7, Rd8, Rd9)

According to Table 1, the anthropogenic activities at Rd5 are mainly associated with motor vehicle related businesses such as car servicing and petrol stations while the primary anthropogenic activities at Rd3 


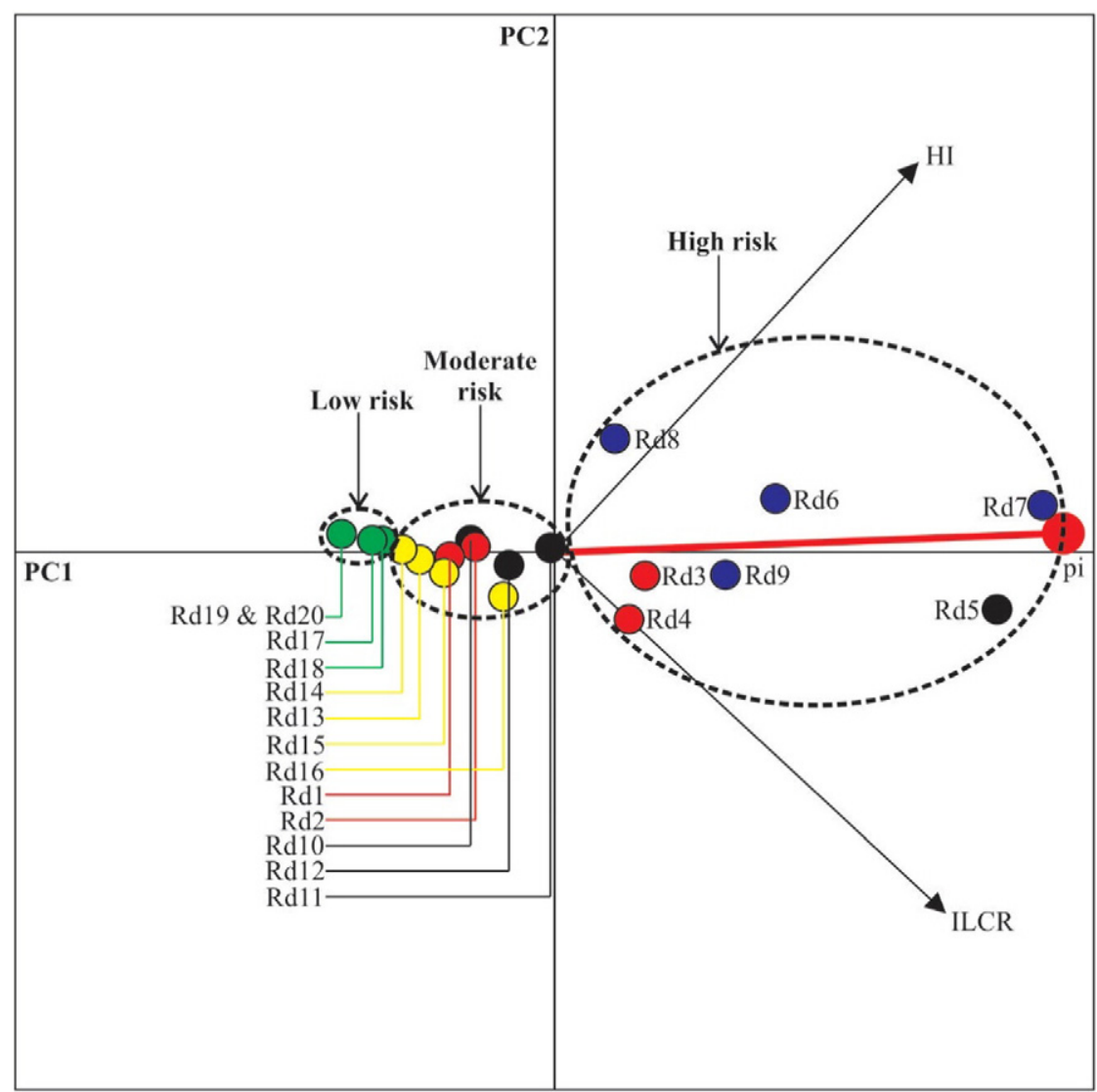

Fig. 3. GAIA biplot of the relationship between risk arising from PAHs and TMs with the study sites

and $\mathrm{Rd} 4$ are retail, education, catering, hospitality and commercial offices. The former anthropogenic activities contribute higher loadings of TMs and PAHs to stormwater compared to the latter (Onianwa et al., 2003; Guo et al., 2012), resulting in higher concentration and risk of TMs and PAHs in stormwater (Fig. 3). The high risk posed by TMs and PAHs in stormwater from Rd3 and Rd4 is due to the high traffic congestion which is attributed to the traffic lights installed at the road intersections and numerous car parking bays along the roadside. The high traffic congestion around Rd3 and Rd4 can lead to frequent braking and starting activities which in turn contribute high loadings and risk of TMs and PAHs in stormwater due to increased brake and tyre wear (Amato et al., 2009; Luo et al., 2010). The main anthropogenic activities at Rd6, Rd7, Rd8 and Rd9 are various light industrial activities which also generate high loads of TMs and PAHs (Bruschweiler et al., 2012; Kho et al., 2014).

Table 2

Characteristics of the anthropogenic activities and traffic congestion related to different risk levels.

\begin{tabular}{|c|c|c|}
\hline Anthropogenic activities & $\begin{array}{l}\text { Traffic } \\
\text { congestion }\end{array}$ & Risk level \\
\hline Industrial businesses & High & High risk \\
\hline Motor vehicle related businesses & High & High risk \\
\hline \multirow{2}{*}{$\begin{array}{l}\text { Retail, education, hospitality, catering and } \\
\text { commercial offices }\end{array}$} & High & High risk \\
\hline & Low & $\begin{array}{l}\text { Moderate } \\
\text { risk }\end{array}$ \\
\hline $\begin{array}{l}\text { Residential activities mixed with commercial } \\
\text { businesses }\end{array}$ & Low & $\begin{array}{l}\text { Moderate } \\
\text { risk }\end{array}$ \\
\hline Only residential activities & Low & $\begin{array}{l}\text { Moderate } \\
\text { risk }\end{array}$ \\
\hline Very limited anthropogenic activities & Low & Low risk \\
\hline
\end{tabular}

Table 3

Anthropogenic activities and traffic characteristics of the study sites.

\begin{tabular}{|c|c|c|c|c|c|}
\hline $\begin{array}{l}\text { Study } \\
\text { sites }\end{array}$ & $\begin{array}{l}\text { Motor } \\
\text { vehicle } \\
\text { related } \\
\text { businesses } \\
\text { (FVS) }\end{array}$ & $\begin{array}{l}\text { Retail, } \\
\text { education, } \\
\text { catering and } \\
\text { office } \\
\text { businesses } \\
\text { (FCS) }\end{array}$ & $\begin{array}{l}\text { Residential } \\
\text { activities mixed } \\
\text { with commercial } \\
\text { businesses (RMC) }\end{array}$ & $\begin{array}{l}\text { Only } \\
\text { residential } \\
\text { activities } \\
(\mathrm{RON})\end{array}$ & $\begin{array}{l}\text { Traffic } \\
\text { congestion } \\
(\mathrm{TC})\end{array}$ \\
\hline Rd1 & 0 & 2 & 0 & 0 & 0 \\
\hline $\mathrm{Rd} 2$ & 0 & 2 & 0 & 0 & 0 \\
\hline $\mathrm{Rd} 3$ & 0 & 2 & 0 & 0 & 1 \\
\hline $\mathrm{Rd} 4$ & 0 & 2 & 0 & 0 & 1 \\
\hline Rd5 & 1 & 0 & 0 & 0 & 1 \\
\hline Rd6 & 1 & 0 & 0 & 0 & 1 \\
\hline $\mathrm{Rd} 7$ & 1 & 0 & 0 & 0 & 1 \\
\hline $\mathrm{Rd} 8$ & 0 & 0 & 0 & 0 & 0 \\
\hline Rd9 & 0 & 0 & 0 & 0 & 0 \\
\hline $\operatorname{Rd} 10$ & 0 & 1 & 1 & 0 & 0 \\
\hline Rd11 & 0 & 1 & 1 & 0 & 0 \\
\hline $\mathrm{Rd} 12$ & 0 & 1 & 1 & 0 & 0 \\
\hline Rd13 & 0 & 0 & 0 & 1 & 0 \\
\hline Rd14 & 0 & 0 & 0 & 1 & 0 \\
\hline Rd15 & 0 & 0 & 0 & 1 & 0 \\
\hline Rd16 & 0 & 0 & 0 & 1 & 0 \\
\hline Rd17 & 0 & 0 & 0 & 0 & 0 \\
\hline Rd18 & 0 & 0 & 0 & 0 & 0 \\
\hline Rd19 & 0 & 0 & 0 & 0 & 0 \\
\hline $\mathrm{Rd} 20$ & 0 & 0 & 0 & 0 & 0 \\
\hline
\end{tabular}

Note: 1/0 of FVS - existing/non-existing motor vehicle related businesses; $2 / 1 / 0$ of FCS high/low/non fraction of retail, education, hospitality, catering and office businesses (high fraction in commercial areas while low fraction in residential areas); 1/0 of RMC existing/non-existing commercial businesses in residential areas; $1 / 0$ of RON - only residential houses/residential houses mixed with commercial businesses; $1 / 0$ of TC - high/ low traffic congestion. 


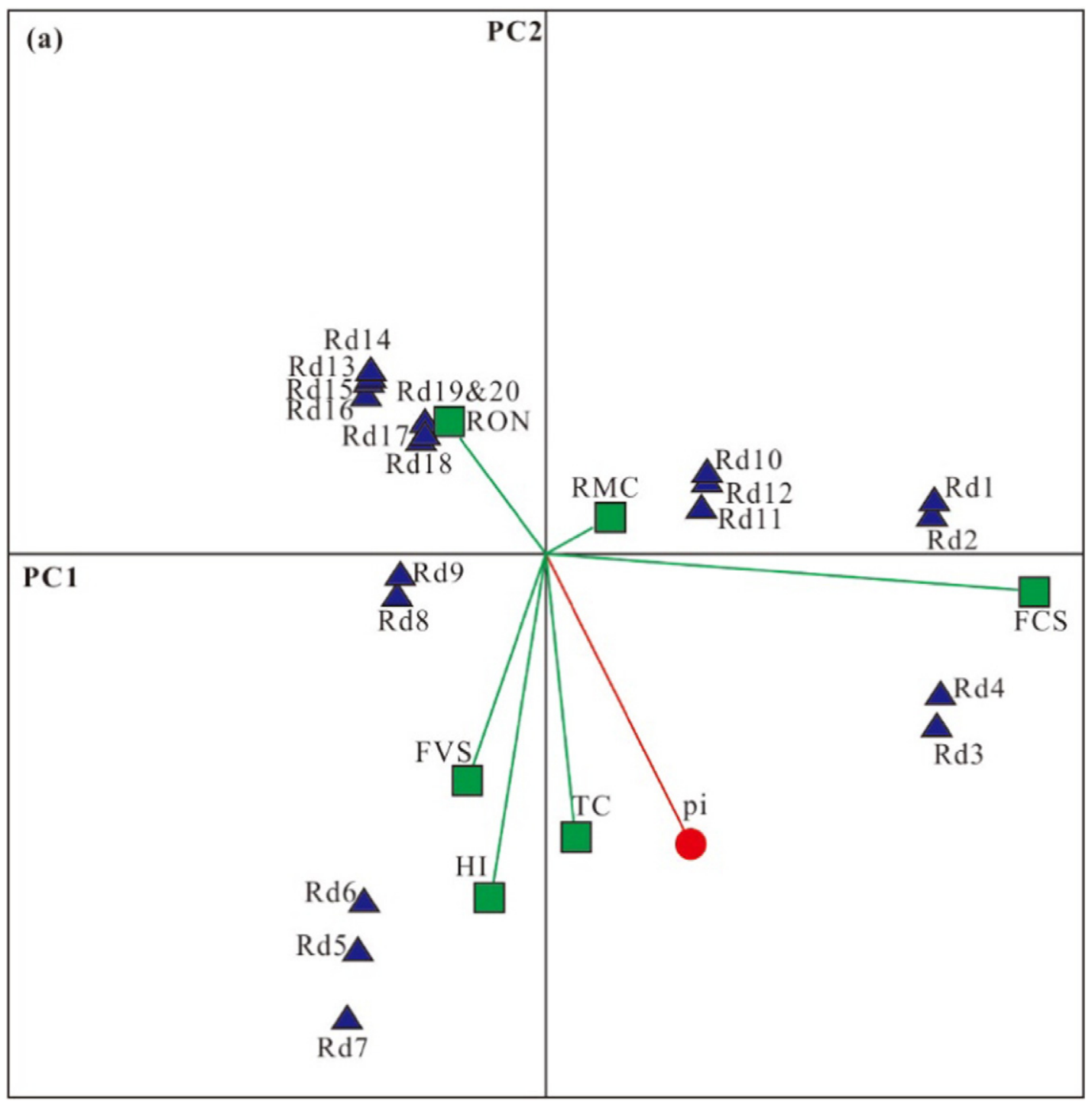

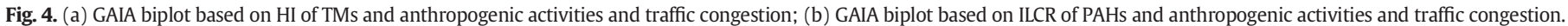

(b) Moderate risk (Rd1, Rd2, Rd10, Rd11, Rd12, Rd13, Rd14, Rd15, Rd16)

As evident in Table 1, the anthropogenic activities at $\mathrm{Rd} 1, \mathrm{Rd} 2, \mathrm{Rd} 3$ and $\mathrm{Rd} 4$ are the same. However, the traffic congestion around $\mathrm{Rd} 1$ and $\mathrm{Rd} 2$ is relatively lower compared to the intense traffic around $\mathrm{Rd} 3$ and Rd4. Consequently, the risk arising from TMs and PAHs in stormwater from Rd 1 and $\mathrm{Rd} 2$ is moderate. According to Fig. 3, although the risks posed by the pollutants in stormwater from Rd10, Rd11, Rd12, Rd13, Rd14, Rd15 and Rd16 are all categorised at the moderate level, the risks from stormwater from $\mathrm{Rd} 10, \mathrm{Rd} 11$ and $\mathrm{Rd} 12$ are overall higher than that from Rd13, Rd14, Rd15 and Rd16. As shown in Table 1, the anthropogenic activities around road sites Rd10, Rd11 and Rd12 are residential activities mixed with a range of commercial premises such as retail, hospitality, catering and education while the anthropogenic activities around the latter road sites are only residential activities. Due to the relatively higher loads of TMs and PAHs generated from commercial activities compared to residential activities (Gunawardana et al., 2011), the risk associated with stormwater from Rd10, Rd11 and Rd12 is generally higher than that from $\mathrm{Rd} 13, \mathrm{Rd} 14, \mathrm{Rd} 15$ and $\mathrm{Rd} 16$. This explains the relative positions of these sites along the PC1 axis in Fig. 3. (c) Low risk (Rd17, Rd18, Rd19, Rd20)

The anthropogenic activities at $\mathrm{Rd} 17, \mathrm{Rd} 18, \mathrm{Rd} 19$ and $\mathrm{Rd} 20$ are sparse as these sites are located in natural land use areas and the traffic congestion is very low. Due to the low generation of TMs and PAHs, the risk posed by these pollutants in stormwater is at the lowest. Similar results were found by Peng et al. (2016) who investigated the variation of risk posed by PAHs in soils from areas with different urbanisation levels.

(d) Summary of analysis outcomes

Based on the reasons attributed for the risk levels, the characteristics of the anthropogenic activities and traffic congestion are summarised in Table 2. As evident from Table 2, the risk to human health from TMs and PAHs in stormwater from industrial sites are all categorised at the high risk level. The TMs and PAHs in stormwater from all natural sites pose low risk to human health. This indicates that stormwater from industrial and natural land use areas is influenced by anthropogenic activities and traffic congestion only to a minor extent. On the other hand, the risk posed by TMs and PAHs in stormwater from commercial and residential 


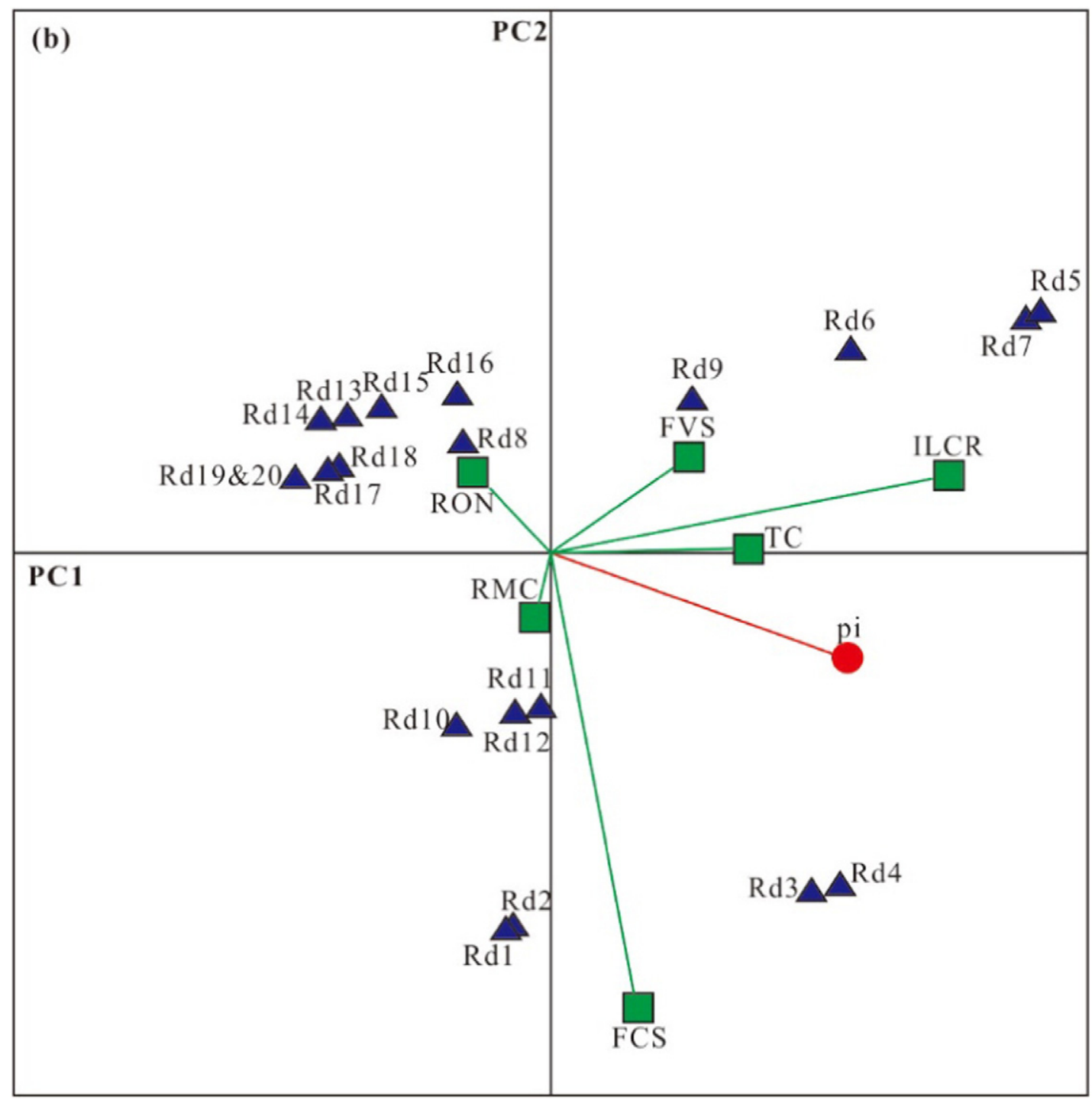

Fig. 4 (continued).

areas is significantly influenced by anthropogenic activities and traffic congestion.

The commercial land use activities can be categorised into two main anthropogenic activities taking place; motor vehicle related businesses (e.g. Rd5); and retail, education, hospitality, catering and commercial offices (e.g. Rd1, Rd2, Rd3 and Rd4). The former anthropogenic activities generate stormwater runoff with higher risks from TMs and PAHs than the latter. Furthermore, traffic congestion could potentially be an influential factor which impacts on the risk from the latter anthropogenic activities. Higher traffic congestion leads to relatively higher risk from TMs and PAHs in stormwater. The residential land use activities also have two primary anthropogenic activity categories, namely, residential activities mixed with commercial businesses (e.g. Rd10, Rd11 and Rd12) and only residential activities (e.g. Rd13, Rd14, Rd15 and Rd16). The risks from TMs and PAHs in the stormwater from the former sites are higher than the latter.

\subsection{Re-formulating the risk model}

3.3.1. Selection of anthropogenic activities and traffic congestion factors to assess risk

To include anthropogenic activities and traffic congestion in quantitative risk assessment of TMs and PAHs in stormwater, Eq. (1) was re- formulated. Accordingly, appropriate factors to represent the relevant anthropogenic activities and traffic congestion needed to be selected. Reference to the discussion in Section 3.2, the factors applicable to the study sites are listed in Table 3.

The selection of appropriate factors in Table 3 was conducted using the PROMETHEE and the GAIA methods. Two data matrices with 20 actions and 6 criteria were employed to derive the GAIA biplots. One data matrix comprised of 20 study sites $\times$ HI, FVS, FCS, RMC, RON and TC and the other comprised of 20 study sites $\times$ ILCR FVS, FCS, RMC, RON and TC. The results of GAIA analysis are given in Fig. 4. It can be seen from Fig. 4(a) that the vectors for fraction of motor vehicle related businesses (FVS) and traffic congestion (TC) form acute angles with HI and this observation is also evident in Fig. 4(b). This means that the fraction of motor vehicle related businesses and traffic congestion is closely relate to the risk from TMs and PAHs in stormwater. However, the risk indices have a relatively limited relationship with other factors including RMC, RON and TC. Therefore, FVS and TC should be considered to evaluate risk from TMs and PAHs in stormwater.

\subsubsection{Re-formulation of the risk assessment procedure}

Taking into consideration the two factors relating to anthropogenic activities and traffic congestion, risk posed by TMs and PAHs in stormwater can be defined by Eq. (2). The risk assessment is briefly 
Table 4

Results from the concentration assessment of pollutants in stormwater.

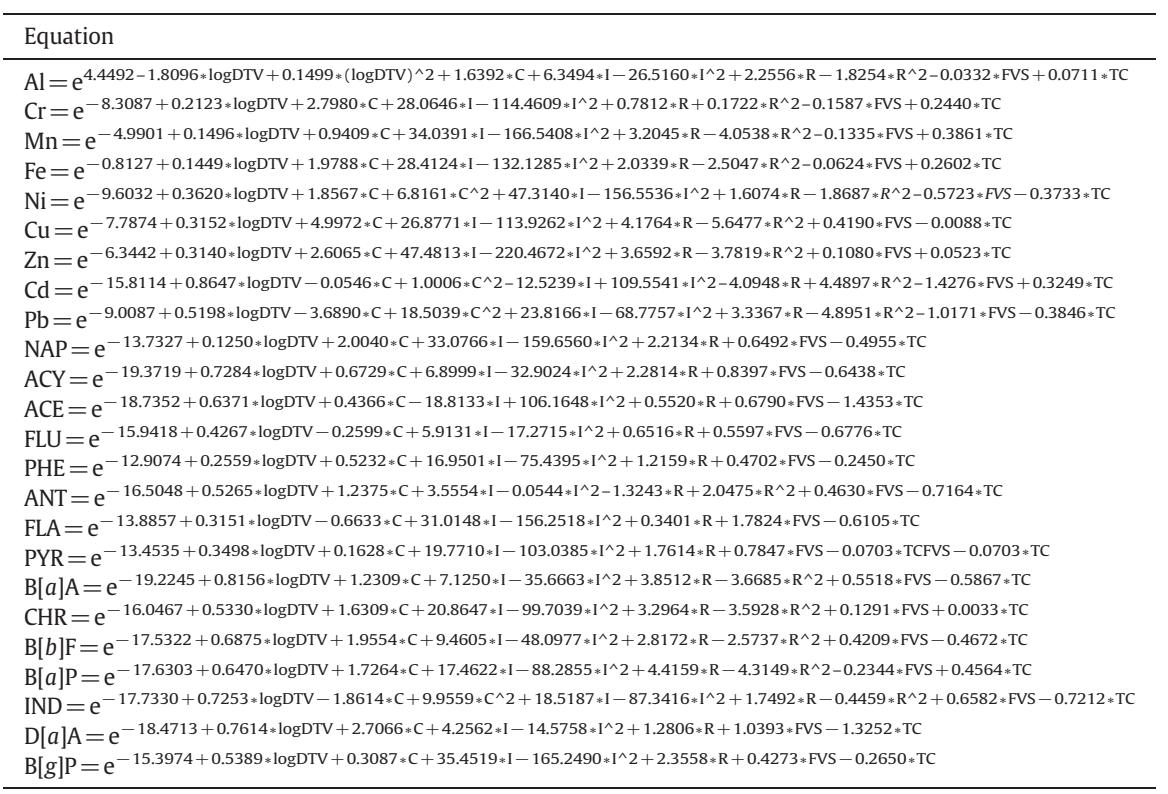

discussed below and detailed information can be found in Ma et al (2016). Firstly, the concentration of TMs and PAHs was modelled using the geoR package in R software based on the Matern covariance function (Diggle and Ribeiro, 2007). The concentrations were modelled as a function of land use (C, I and R), daily traffic volume (DTV), motor vehicle related businesses (FVS) and traffic congestion (TC). The values of FVS and TC were derived from Table 3 . The modelling results for estimating concentrations of TMs and PAHs are shown in Table 4. The quadratic terms in the equations account for the curved relationship observed between the residual and the independent variable. The trend of the residual variability against the independent variables was excluded. An example of residual variability is shown in Fig. S1 and the estimates and standard errors are summarised in Table S7 in the Supplementary Information. Secondly, the risk from TMs and PAHs in stormwater was assessed based on the risk assessment methodology including toxicity identification, exposure assessment, dose-response assessment and risk characterisation (USEPA, 1989).

Risk $=f(\mathrm{DTV}, \mathrm{C}, \mathrm{I}, \mathrm{R}, \mathrm{FVS}, \mathrm{TC})$

where: DTV - daily traffic volume [vehicles per day];

$\mathrm{C}, \mathrm{I}$ and $\mathrm{R}$ - percentage of commercial, industrial and residential land use areas within $1 \mathrm{~km}$ around the site;

FVS - motor vehicle related businesses;

TC - traffic congestion.

The improved risk model derived can be found in Eqs. (3) and (4).

$\mathrm{HI}=0.05 \times \mathrm{Al}+68.20 \times \mathrm{Cr}+2.70 \times \mathrm{Mn}+0.02 \times \mathrm{Fe}+1.33 \times \mathrm{Ni}$

$+0.63 \times \mathrm{Cu}+0.10 \times \mathrm{Zn}+409.18 \times \mathrm{Cd}+10.83 \times \mathrm{Pb}$

Where: The abbreviations of TMs are derived from Table 4.

$$
\begin{aligned}
\mathrm{ILCR}= & \frac{1.190789424}{243236} \times 0.001 \times \mathrm{NAP}+0.001 \times \mathrm{ACY}+0.001 \times \mathrm{ACE} \\
& +0.001 \times \mathrm{FLU}+0.001 \times \mathrm{PHE}+0.01 \times \mathrm{ANT}+0.001 \\
& \times \mathrm{FLA}+0.001 \times \mathrm{PYR}+0.1 \times \mathrm{B}[a] \mathrm{A}+0.01 \times \mathrm{CHR}+0.1 \\
& \times \mathrm{B}[b] \mathrm{F}+1 \times \mathrm{B}[a] \mathrm{P}+0.1 \times \mathrm{IND}+5 \times \mathrm{D}[a] \mathrm{A}+0.01 \\
& \times \mathrm{B}[g] \mathrm{P}
\end{aligned}
$$

Where: The abbreviations of PAHs are derived from Table 4 .

The accuracy of the model was validated using the leave-one-out cross-validation approach (Wold, 1978). The predicted data on pollutant concentration in stormwater was verified to be similar when compared with the corresponding observed data. An example of the comparison of predicted concentration of $\mathrm{Al}$ in stormwater from Rd1 when Rd1 was left out from the data set with the observed concentrations of Al at Rd1 is shown in Fig. S2 in the Supplementary Information.

\subsection{Stormwater management based on improved understanding of human health risk}

Since anthropogenic activities and traffic congestion were found to exert influence on the risk associated with stormwater, stormwater management needs to take these factors into consideration. For example, braking and starting activities due to traffic congestion are a primary reason leading to high risk from stormwater. An option would be to reduce traffic congestion and to explore alternate road designs in order to reduce frequent braking and starting activities. For instance, the multi-objective transit network design and frequency setting problem (TNDFSP) discussed by Arbex and da Cunha (2015) aims to design various routes according to demand to reduce traffic congestion in urban areas. In addition, since industrial and motor vehicle related activities contribute to high risk related to stormwater, appropriate stormwater treatment methods such as Water Sensitive Urban Design (WSUD) need to be implemented in areas where these activities are

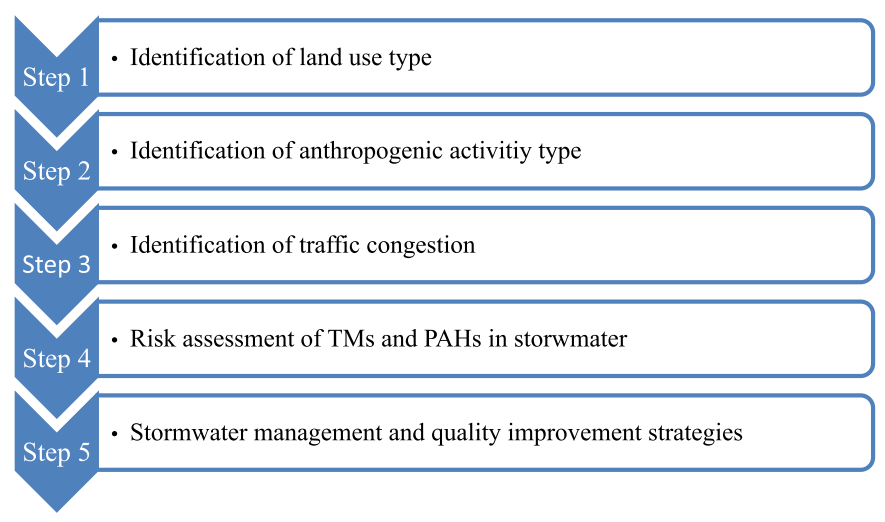

Fig. 5. Roadmap of the approach to human health risk assessment and management of TMs and PAHs in stormwater. 


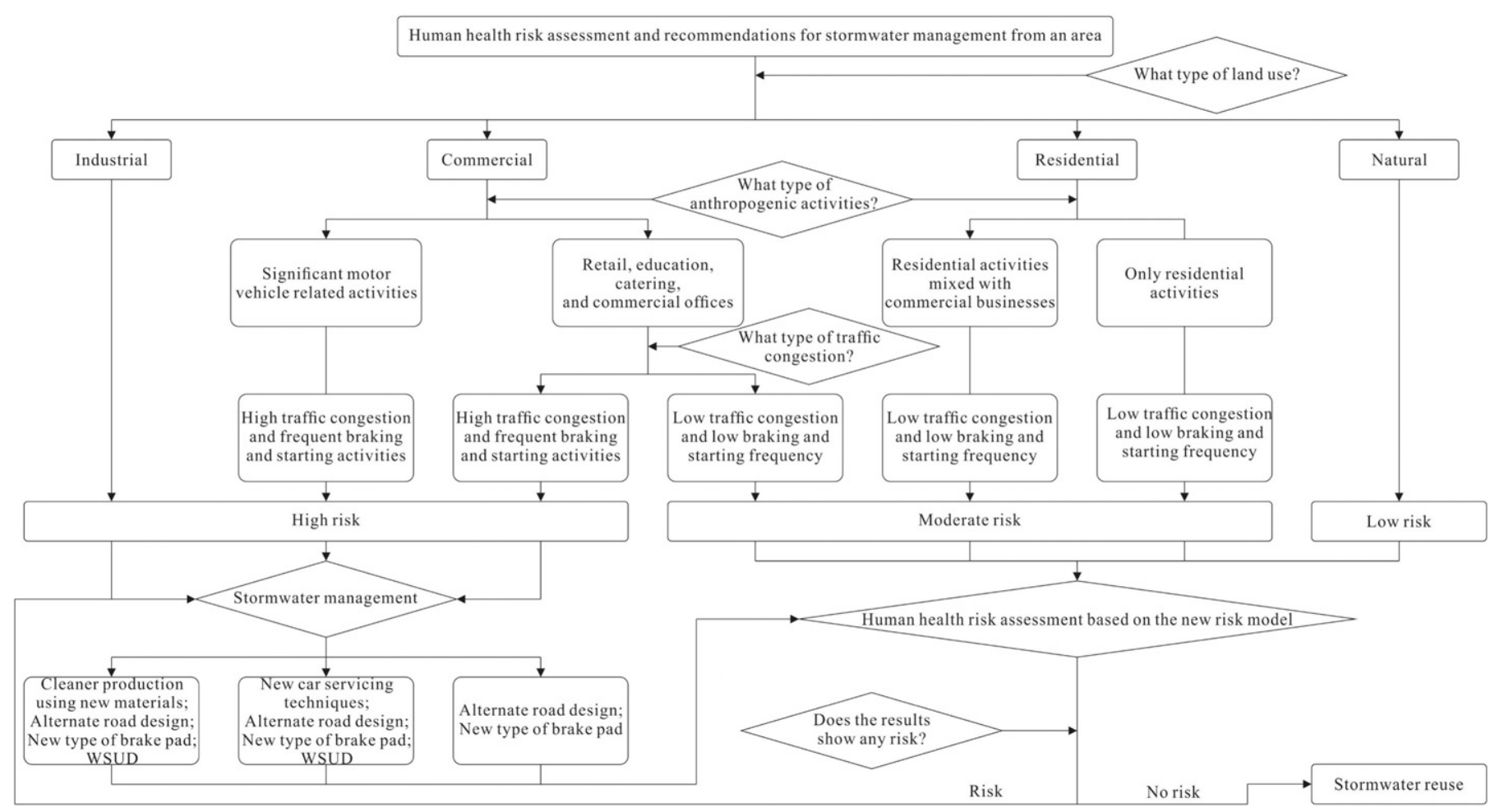

Fig. 6. Flowchart for human health risk assessment and management of the toxic chemical pollutants in stormwater.

prevalent. Technologies for removing TMs and PAHs such as the provision of infiltration systems, treatment wetlands and retention ponds have been investigated in numerous research studies (Hatt et al., 2007; DiBlasi et al., 2008). Furthermore, cleaner production in industrial manufacturing and new techniques in vehicle servicing need to be investigated to reduce the generation of TMs and PAHs (Chen et al., 2015). Accordingly, a generic roadmap of the approach for the assessment and management of human health risk arising from TMs and PAHs in stormwater can be developed as illustrated in Fig. 5. This roadmap is generic and can be applied at any geographic location.

Based the roadmap shown in Fig. 5, a flowchart illustrating the approach for the robust assessment and management of human health risk from TMs and PAHs in stormwater is summarised in Fig. 6 which again is applicable to any geographic area. For example, if an area is identified as a commercial land use area, the anthropogenic activities and traffic congestion features need to be further defined due to influence they exert on the risk from stormwater. Stormwater with high risk need to be managed using appropriate methods. The risk from stormwater can be estimated using Eqs. (3) and (4) to determine whether the stormwater is safe to be reused for recreational and potable purposes or needs further treatment. It is important to note that this flowchart has been designed to only assess and manage the risk associated with TMs and PAHs in stormwater and excludes other pollutants that may also be present. The methodology developed as depicted in the roadmap in Fig. 5 and the flowchart in Fig. 6 can be further extended to assess and manage risk to human health posed by other stormwater pollutants or for different land use areas.

\section{Conclusions}

The research study confirmed that anthropogenic activities and traffic congestion need to be taken into consideration to derive a more accurate understanding of the risk to human health from TMs and PAHs in urban stormwater. Anthropogenic activities are an influential factor on the risk arising from pollutants in stormwater from commercial and residential areas. The commercial land use activities can be categorised into two main anthropogenic activities; motor vehicle related businesses; and retail, education, catering, hospitality and commercial offices. The former anthropogenic activities generate stormwater runoff with relatively higher risks from TMs and PAHs than the latter. The residential land use activities also have two primary categories of anthropogenic activities, namely, residential activities mixed with commercial businesses and only residential activities. Risks from TMs and PAHs in the stormwater from the former area are higher than the latter.

\section{Appendix A. Supplementary data}

Supplementary data to this article can be found online at http://dx. doi.org/10.1016/j.scitotenv.2016.11.015.

\section{References}

Amato, F., Pandolfi, M., Viana, M., Querol, X., Alastuey, A., Moreno, T., 2009. Spatial and chemical patterns of PM10 in road dust deposited in urban environment. Atmos. Environ. 43 (9), 1650-1659.

Arbex, R.O., Cunha, C.B.D., 2015. Efficient transit network design and frequencies setting multi-objective optimization by alternating objective genetic algorithm. Transport Res B-Meth. 81, 355-376

Begum, S., Rasul, M., Brown, R.J., 2008. Stormwater treatment and reuse techniques: a review. Trans. Environ. Dev. 4 (11), 144-149.

Bruschweiler, E.D., Danuser, B., Huynh, C.K., Wild, P., Schupfer, P., Vernez, D., Boiteux, P., Hopf, N.B., 2012. Generation of polycyclic aromatic hydrocarbons (PAHs) during woodworking operations. Front. Oncol. 2.

Chen, S.-C., Liao, C.-M., 2006. Health risk assessment on human exposed to environmental polycyclic aromatic hydrocarbons pollution sources. Sci. Total Environ. 366 (1), 112-123.

Chen, Y., Santos, A., Ho, D., Wang, Y., Kumeria, T., Li, J., Wang, C., Losic, D., 2015. On the generation of interferometric colors in high purity and technical grade aluminum: an alternative green process for metal finishing industry. Electrochim. Acta 174, 672-681.

DiBlasi, C.J., Li, H., Davis, A.P., Ghosh, U., 2008. Removal and fate of polycyclic aromatic hydrocarbon pollutants in an urban stormwater bioretention facility. Environ. Sci. Technol. 43 (2), 494-502.

Diggle, P., Ribeiro, P.J., 2007. Model-based Geostatistics. Springer Science \& Business Media, New York. 
Egodawatta, P., Goonetilleke, A., 2006. Characteristics of Pollutants Built-up on Residentia Road Surfaces. Proceedings of the 7th International Conference on HydroScience and Engineering (Philadelphia, U.S.A.).

Egodawatta, P., Thomas, E., Goonetilleke, A., 2007. Mathematical interpretation of pollutant wash-off from urban road surfaces using simulated rainfall. Water Res. 41 (13), 3025-3031.

Gunawardana, C., Egodawatta, P., Goonetilleke, A., 2014. Role of particle size and composition in metal adsorption by solids deposited on urban road surfaces. Environ. Pollut. $184,44-53$.

Gunawardana, C., Goonetilleke, A., Egodawatta, P., Dawes, L., Kokot, S., 2011. Role of solids in heavy metals buildup on urban road surfaces. J. Environ. Eng. 138 (4), 490-498.

Gunawardana, C.T.K., 2011. Influence of Physical and Chemical Properties of Solids on Heavy Metal Adsorption. Queensland University of Technology, Australia (PhD Degree).

Gunawardena, J., Egodawatta, P., Ayoko, G.A., Goonetilleke, A., 2012. Role of traffic in atmospheric accumulation of heavy metals and polycyclic aromatic hydrocarbons. Atmos. Environ. 54, 502-510.

Gunawardena, J., Egodawatta, P., Ayoko, G.A., Goonetilleke, A., 2013. Atmospheric deposition as a source of heavy metals in urban stormwater. Atmos. Environ. 68, 235-242.

Guo, G., Wu, F., Xie, F., Zhang, R., 2012. Spatial distribution and pollution assessment of heavy metals in urban soils from southwest China. J. Environ. Sci. 24 (3), 410-418.

Hatt, B.E., Deletic, A., Fletcher, T.D., 2007. Stormwater reuse: designing biofiltration systems for reliable treatment. Water Sci. Technol. 55 (4), 201-209.

Herngren, L., Goonetilleke, A., Ayoko, G.A., 2006. Analysis of heavy metals in road-deposited sediments. Anal. Chim. Acta 571 (2), 270-278.

Herngren, L.F., 2005. Build-up and Wash-off Process Kinetics of PAHs and Heavy Metals on Paved Surfaces Using Simulated Rainfall. Queensland University of Technology, Australia (PhD Degree).

Järup, L., 2003. Hazards of heavy metal contamination. Br. Med. Bull. 68 (1), 167-182.

Kho, Y., Lee, E.-H., Chae, H.J., Choi, K., Paek, D., Park, S., 2014. 1-hydroxypyrene and oxidative stress marker levels among painting workers and office workers at shipyard. Int. Arch. Occup. Environ. Health 88, 297-303.

Lee, S.-W., Hwang, S.-J., Lee, S.-B., Hwang, H.-S., Sung, H.-C., 2009. Landscape ecological approach to the relationships of land use patterns in watersheds to water quality characteristics. Landsc. Urban Plan. 92 (2), 80-89.

Liu, A., Goonetilleke, A., Egodawatta, P., 2012. Inadequacy of land use and impervious area fraction for determining urban stormwater quality. Water Resour. Manag. 26 (8), 2259-2265

Liu, A., Gunawardana, C., Gunawardena, J., Egodawatta, P., Ayoko, G.A., Goonetilleke, A. 2016a. Taxonomy of factors which influence heavy metal build-up on urban road surfaces. J. Hazard. Mater. 310, 20-29.
Liu, L., Liu, A., Li, Y., Zhang, L., Zhang, G., Guan, Y., 2016b. Polycyclic aromatic hydrocarbons associated with road deposited solid and their ecological risk: implications for road stormwater reuse. Sci. Total Environ. 563-564, 190-198.

Luo, Z., Li, Y., Nazaroff, W.W., 2010. Intake fraction of nonreactive motor vehicle exhaust in Hong Kong. Atmos. Environ. 44 (15), 1913-1918.

Ma, Y., 2016. Human Health Risk of Toxic Chemical Pollutants Generated From Traffic and Land Use Activities. Queensland University of Technology, Australia (PhD Degree).

Ma, Y, Egodawatta, P. McGree, J Liu, A Goonetilleke, A 2016. Human health risk assessment of heavy metals in urban stormwater. Sci. Total Environ. 557-558, 764-772.

Makepeace, D.K., Smith, D.W., Stanley, S.J., 1995. Urban stormwater quality: summary of contaminant data. Crit. Rev. Environ. Sci. Technol. 25 (2), 93-139.

NHMRC\&AWRC, 2011. Australian Drinking Water Guidelines. Australian Government Publishing Service, Canberra .

Onderka, M., Wrede, S., Rodný, M., Pfister, L., Hoffmann, L., Krein, A., 2012. Hydrogeologic and landscape controls of dissolved inorganic nitrogen (DIN) and dissolved silica (DSi) fluxes in heterogeneous catchments. J. Hydrol. S 450-451 (2), 36-47.

Onianwa, P., Jaiyeola, O., Egekenze, R., 2003. Heavy metals contamination of topsoil in the vicinities of auto-repair workshops, gas stations and motor-parks in a Nigerian city. Toxicol. Environ. Chem. 84 (1-4), 33-39.

Peng, C., Wang, M., Zhao, Y., Chen, W., 2016. Distribution and risks of polycyclic aromatic hydrocarbons in suburban and rural soils of Beijing with various land uses. Environ. Monit. Assess. 188 (3), 1-12.

Sansalone, J.J., Buchberger, S.G., 1997. Partitioning and first flush of metals in urban roadway storm water. J. Environ. Eng. 123 (2), 134-143.

USEPA, 1984. Guidelines Establishing Test Procedures for the Analysis of Pollutants Under Clean Water Act: Method 610 - Polynuclear Aromatic Hydrocarbons. 49. US Environmental Protection Agency, Washington, DC, pp. 43344-43352.

USEPA, 1989. Risk Assessment Guidance for Superfund, Volume I, Human Health Evaluation Manual (Part A). US Environmental Protection Agency, Washington, DC

USEPA, 1994. Metod 200.8: Trace Elements in Waters and Wastes by Inductively Coupled Plasma-Mass Spectrometry. US Environmental Protection Agency, Washington, DC.

USEPA, 1996. Pressurized Fluid Extraction (PFE). US Environmental Protection Agency, Washington, DC

Vaze, J., Chiew, F.H.S., 2002. Experimental study of pollutant accumulation on an urban road surface. Urban Water 4 (4), 379-389.

Wold, S., 1978. Cross-validatory estimation of the number of components in factor and principal components models. Technometrics 20 (4), 397-405. 